\title{
Arbitrary High Order Schemes for Transport Problems
}

\author{
B. Latorre ${ }^{1} \&$ P. García-Navarro ${ }^{2}$ \\ ${ }^{1}$ Soil and Water Department. Estación Experimental de Aula Dei. CSIC. Spain. \\ ${ }^{2}$ Fluid Mechanics. University of Zaragoza. Spain.
}

In this work, a method based on Legendre polynomials is presented for the simulation of the passive transport of a solute. The formulation is conservative, explicit and made in a single step. The spatial accuracy is achieved by means of cell polynomial approximations using Legendre series. This kind of spatial representation is also found in finite element discretization and allows for information on the variation of the fields at the sub-grid scale. The time resolution of the transport is based on both a numerical estimation of the displacement at the advection speed and a grid deformation, according to the semi-Lagrangian rules, followed by a projection of the solution on the fixed initial grid.

First, the resolution of the scalar transport of a concentration field is presented. The main interest is focused on the analysis of the accuracy and the efficiency of the method when moving from order 1 to order 20 as compared to standard methods of virtual reconstruction. The interest in this work is the study of the computational saving that can be achieved if the required accuracy is medium or low. This is possible thanks to the sub-grid information that offers the possibility to solve problems with enough accuracy using only a few grid cells and high order polynomials. Furthermore, this enables the use of large time steps hence leading to low computational times.

In a second part, the method is applied to the resolution of the passive transport of a solute in shallow water flows. A technique has been developed to couple Legendre schemes to any conservative method used for the resolution of the shallow water equations. The coupling offers the possibility to combine solvers of different order of accuracy, always enforcing conservative and monotone behavior in the numerical solution of the solute concentration. This strategy is interesting since it is possible to require high order of accuracy only in the solute transport simulation, hence concentrating the computational effort in the component with more numerical error. The coupled method is applied to solve transport problems including bed level variations (source terms in the flow equations), water depth discontinuities and different regimes in order to analyze the performance of the proposed coupling technique.

\section{INTRODUCTION}

This work aims to develop and study efficient tools for the simulation of the transport and mixing of a passive solute in free surface flows.

The numerical resolution of passive transport is more demanding than the calculation of water flow. This phenomenon is justified from different perspectives. The characteristic space (Rusanov 1963) associated to pressure waves that dominate the water flow is compressive presenting certain numerical advantages such as the possibility to obtain good numerical results with a first order approximation. However, passive transport takes place in degenerate characteristic fields. Furthermore, water flow is pseudosteady in most of the problems whereas transport processes are usually transient. Hence, the requirement on the order of approximation increases at least to second order (Bermudez and Vázquez-Cendón 1994, Vázquez-Cendón 1999).
For these reasons, first-order methods that have been used successfully for the simulation of the shallow water equations lead to high errors and low convergence when solving passive transport. It is therefore necessary to develop specific strategies that provide an accurate and efficient resolution of this process.

It is widely accepted that high order methods are an efficient choice when low errors are required in the solutions (Dumbser and Kaeser 2007). Among the different methodologies available for the development of high order methods, we focus on the finite volume formulation. A new family of methods, based on polynomial sub-cell representation, is presented and its properties are analyzed and compared with the ADER (Toro, Millington, and Nejad 2001) methodology. For this purpose, different problems are numerically solved studying the dependence of the accuracy and efficiency of the methods on the discrete parame- 
ters.

Instead of integrating the resolution of water flow and passive transport in a single method, separate strategies have been developed for the calculation of these two processes applying more precision in the passive transport.

Finally, the coupled resolution of the solute transport and the water flow must comply with certain physical principles: preservation of a constant concentration state and a monotone behavior of the concentration field (Burguete, Murillo, and García-Navarro 2008; Murillo, García-Navarro, and Burguete 2009). new mechanism for coupling the two processes has been developed that respects these conditions. The properties of the technique are verified solving the transport of a passive solute in a complex flow.

\section{LEGENDRE SERIES}

The technique to approximate a function, $\widetilde{\phi}(x)$, as linear combination of Legendre polynomials, $P_{n}(x)$, is presented:

$$
\phi(x)=\sum_{n=1}^{N}{ }^{n} \phi P_{n}(x) \simeq \widetilde{\phi}(x)
$$

where the coefficients, ${ }^{n} \phi$, containing the information of the approximation, are calculated solving the projection of the function over different normalized polynomials (Hilbert 1912):

$$
{ }^{n} \phi=\frac{2 n-1}{2} \int_{-1}^{1} \widetilde{\phi}(x) P_{n}(x) d x
$$

where $[-1,1]$ represents the orthogonality domain of the polynomials.

This technique will be used in the proposed numerical method to represent the solute distribution in each cell and time step, adopting the following notation:

$$
\phi_{i}(x, t)=\sum_{n=1}^{N}{ }^{n} \phi_{i}^{t} P_{n}(x)
$$

where index $i$ refers to the cell and $t$ represents time. The space coordinates are assumed to be adapted, matching the orthogonality interval of the Legendre polynomials and the domain of each cell.

\subsection{Conservative approximation}

Among the different sets of orthogonal polynomials (Chebyshev, Hermite, Jacobi, Laguerre and Legendre) only Legendre polynomials present a constant weight function:

$$
w(x)=1
$$

providing thus a relationship between the first coefficient of the series (1) and the average value of the function:

$$
{ }^{1} \phi=\frac{1}{2} \int_{-1}^{1} \widetilde{\phi}(x) d x
$$

The remaining elements of the Legendre basis, due to the principle of orthogonality, present no net area in the domain and do not contribute to the integral value of the approximate function:

$$
\int_{-1}^{1} P_{n}(x) d x=0 \quad \text { if } n>1
$$

Inserting (5) and (6) in (1) it can be demonstrated that the integral of the approximation matches the integral of the initial function:

$$
\int_{-1}^{1} \phi(x) d x=\int_{-1}^{1} \widetilde{\phi}(x) d x
$$

Equation (7) represents the conservation property of this discretization technique and allows to apply Legendre series in the design of a numerical method without affecting the conservation of the variables.

Finite volume methods describe the problems by means of the average value of the variables in each cell. Therefore, this representation technique can be framed as a first order Legendre series. Higher order techniques in the finite volume formulation apply virtual reconstruction of the variables involving information from other cells. On the other hand, Legendre series develop a local calculation of the variable distribution avoiding the reconstruction step.

Legendre methods were introduced by Latorre et al. (Latorre and García-Navarro 2011) for the resolution of the scalar transport equation. These methods, in which the order of approximation is a parameter, produce spatial variations whose scale is smaller than the cell size. This implies an increase in the computational efficiency compared to high order finite volume methods. Transport resolution is performed computing first, by numerical integration techniques, the movement of the mesh nodes, then stretching and cutting the polynomials contained in each cell. The resulting schemes are explicit and maintain a constant stability criterion not conditioned by the order of approximation. Legendre methods present common characteristics with the discretization in finite elements and the temporal resolution applied in conservative semi-Lagrangian methods.

The Legendre method formulation is presented considering, for simplicity, the transport of a concen- 
tration field in one dimension with constant and positive velocity $(v>0)$ :

$$
\vec{\phi}_{i}^{t+\Delta t}=\boldsymbol{A}(c) \vec{\phi}_{i-1}^{t}+\boldsymbol{B}(c) \vec{\phi}_{i}^{t}
$$

where:

$$
\vec{\phi}_{i}^{t}=\left(\begin{array}{c}
{ }^{1} \phi_{i}^{t} \\
\vdots \\
{ }^{N} \phi_{i}^{t}
\end{array}\right), \quad \boldsymbol{A}=\left(\begin{array}{ccc}
A_{11} & \cdots & A_{1 N} \\
\vdots & \ddots & \vdots \\
A_{N 1} & \cdots & A_{N N}
\end{array}\right)
$$

Scheme (8) represents a set of $N$ equations and contains the influence of the previous state on the solution at each cell through the action of matrices $\boldsymbol{A}$ and $\boldsymbol{B}$ depending on the CFL dimensionless number:

$$
c=\frac{|v| \Delta t}{\Delta x}
$$

The expression of the numerical method (8) in the particular case of the second order approximation is next presented:

$$
\begin{aligned}
& \left(\begin{array}{c}
{ }^{1} \phi_{i}{ }^{t+\Delta t} \\
{ }^{2} \phi_{i}{ }^{t+\Delta t}
\end{array}\right)= \\
& c\left(\begin{array}{cc}
1 & 1-c \\
3(c-1) & c^{2}-3(1-c)^{2}
\end{array}\right)\left(\begin{array}{c}
1 \phi_{i-1}^{t} \\
{ }^{2} \phi_{i-1}^{t}
\end{array}\right)+ \\
& (1-c)\left(\begin{array}{cc}
1 & -c \\
3 c & (1-c)^{2}-3 c^{2}
\end{array}\right)\left(\begin{array}{c}
{ }^{1} \phi_{i}^{t} \\
{ }^{2} \phi_{i}^{t}
\end{array}\right)
\end{aligned}
$$

For any order of approximation, the first row of the Legendre schemes matches the ADER method assuming an arbitrary reconstruction of the variable. The contribution of Legendre methods is the representation and resolution of the remaining degrees of freedom, avoiding the reconstruction step using information from other cells.

\subsection{Case 1: Accuracy and costs}

The accuracy and efficiency of Legendre methods is analyzed and compared with those of the ADER methodology. The transport with constant velocity $v$ $=1$ of a Gaussian profile:

$$
\phi(x)=\exp \left(-(x-0.5)^{2} / 0.02\right)
$$

is considered in a periodic domain of size $x \in[0,1]$. This problem is solved using Legendre and ADER methods of different order of approximation and different domain partitions. The error of the numerical results is characterized using the following expression:

$$
L_{1}=\frac{0.286}{N \Delta x} \sum_{i=1}^{N} \int\left|\phi_{i}(x)-\widetilde{\phi}(x)\right| d x
$$

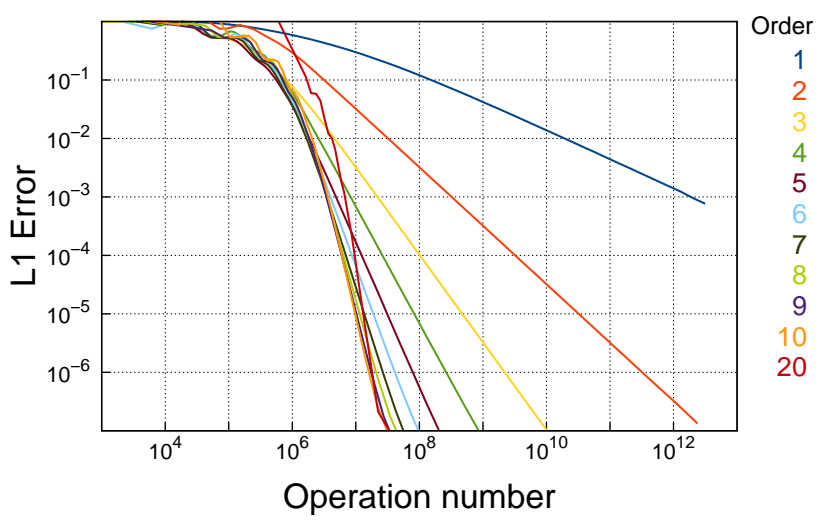

a)

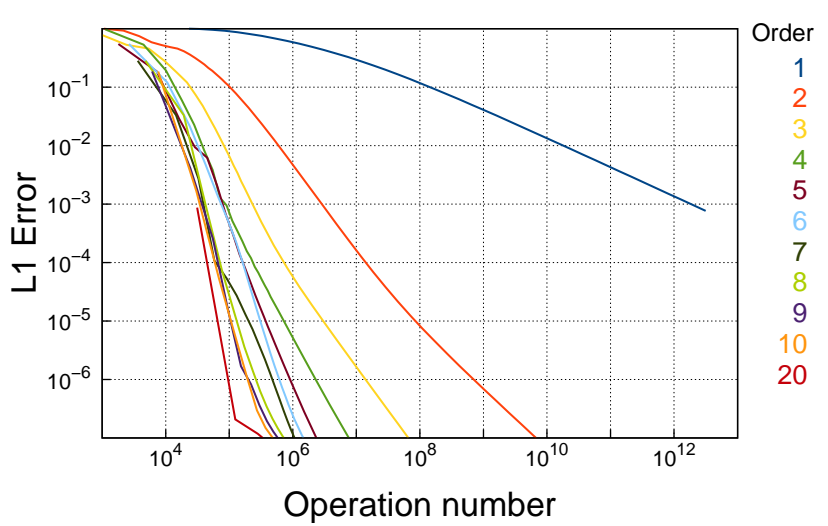

b)

Figure 1: Accuracy and computational costs of ADER a) and Legendre b) methods solving the transport of the Gaussian profile. $(\mathrm{t}=10, \mathrm{CFL}=0.5)$

where $N$ denotes the number of cells, the integral runs along the domain associated with the cell, $\phi_{i}(x)$ represents the numerical results and $\widetilde{\phi}(x)$ the exact solution. The error has been normalized so that the unit value corresponds to the worst result provided by the first order method, which transforms the numerical diffusion profile in a constant state.

The transport of the Gaussian profile is solved during $t=10$ units and a constant time step under $C F L$ $=0.5$ condition. ADER schemes of different order of approximation are first considered using different domain partitions. The error of the results and the number of operations required in each case is represented in logarithmic scale in Figure 1-a. Results from 
the same order of approximation are drawn under the same line, whose value can be found in the legend on the right of the figure. Results of Legendre methods using the same discrete parameters are presented in Figure 1-b.

Figures 1-a and 1-b show that Legendre schemes are more efficient than ADER methods in the resolution of this problem. Comparing the two families for a certain maximum error in the solution, the Legendre methods result 100 times faster than the ADER family for a wide range of errors. Legendre schemes are able to solve transport problems accurately and efficiently using fewer cells and higher orders, situation in which it is not possible to build reconstructions.

\subsection{Case 2: Smolarkiewicz flow}

The propagation of a Gaussian function on a square domain, $x \in[0,6], y \in[0,6]$, under the following velocity field, defined by Smolarkiewicz in 1982 (Smolarkiewicz 1982), is studied:

$$
\left(v_{x}, v_{y}\right)=\frac{\pi}{5}(\sin (\pi x) \sin (\pi y), \cos (\pi x) \cos (\pi y))
$$

This periodic distribution consists of square domains with closed trajectories and alternate senses of rotation. The following initial condition of the concentration field is considered:

$$
\phi(r)=\exp \left(-r^{2} / 0.1\right)
$$

where $r$ represents the distance from the center of the domain:

$$
r=\sqrt{(x-3)^{2}+(y-3)^{2}}
$$

The transport of the initial distribution is solved during two simulation times $(t=5,10)$ using a grid of $120 \times 120$ cells and a constant time step under $C F L=0.5$ condition. Legendre schemes of different order of approximation are considered. The representation of the velocity field and the resolution of the movement of the mesh nodes is made in each method using numerical techniques of the same order of accuracy.

Results obtained using Legendre schemes of order 1, 2 and 3 are shown in Figure 2 using a color map. The Gaussian distribution is transported and deformed in two rotational flows with opposite sense of rotation. The first order scheme presents high numerical diffusion and a divergent calculation of the trajectories, shifting the concentration outside the squares. Theses errors are reduced when considering higher order methods.

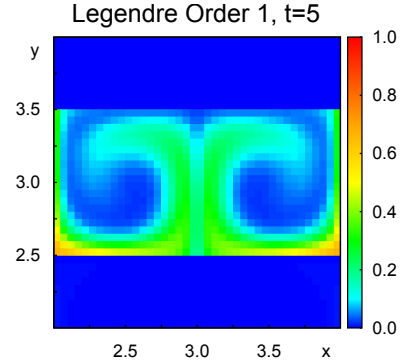

a)

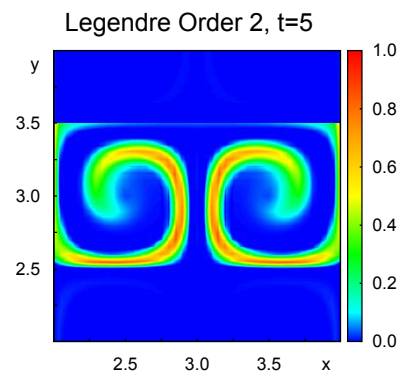

c)

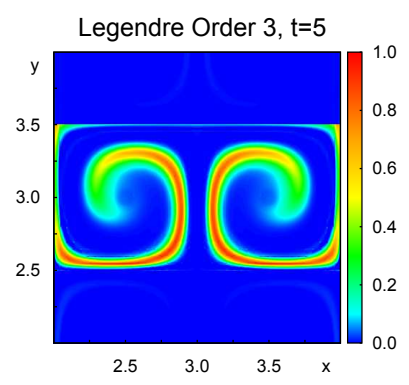

e)

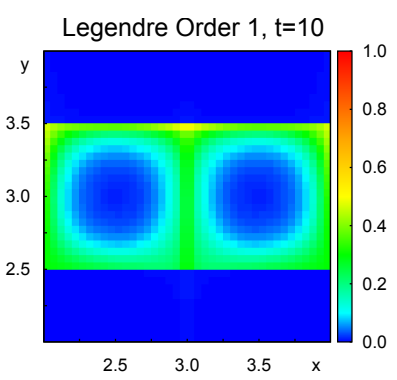

b)

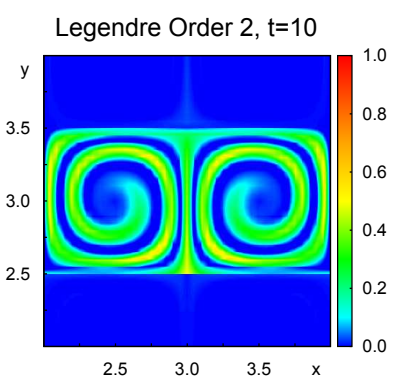

d)

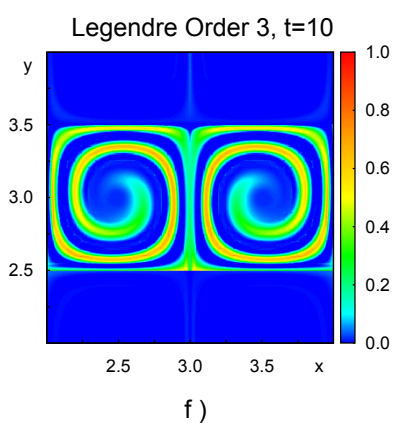

Figure 2: Numerical results corresponding to the transport of the Gaussian profile in the Smolarkiewicz flow using Legendre methods of order 1, 2 and 3. $(\mathrm{t}=(5,10), \mathrm{CFL}=0.5,120 \times 120$ cells $(40 \times 40$ represented)) 


\section{DEPTH-AVERAGED TRANSPORT}

The shallow water model in one dimension, assuming for simplicity a frictionless rectangular channel of unit width, is presented:

$$
\frac{\partial \vec{u}(x, t)}{\partial t}+\frac{\partial \vec{f}(x, t)}{\partial x}=\vec{s}(x)
$$

This system of equations describes the evolution of the variables that characterize the flow in terms of the spatial variations of the physical flux vector $\vec{f}$ and the action of the source terms contained in the vector $\vec{s}$. These terms depend on the water depth $h$, the unit discharge $q$ and the channel bed elevation $z$ as follows:

$$
\vec{u}=\left(\begin{array}{c}
h \\
q
\end{array}\right), \vec{f}=\left(\begin{array}{c}
q \\
\frac{q^{2}}{h}+\frac{g h^{2}}{2}
\end{array}\right), \vec{s}=\left(\begin{array}{c}
0 \\
-g h \frac{\partial z}{\partial x}
\end{array}\right)
$$

This work is focused on explicit numerical methods. In this case, the conservative resolution of the shallow water equations can be formulated using the following general expression:

$$
\vec{u}_{i}^{t+\Delta t}=\vec{u}_{i}^{t}-\frac{\Delta t}{\Delta x}\left(\vec{f}_{i+\frac{1}{2}}-\vec{f}_{i-\frac{1}{2}}-\vec{s}_{i}\right)
$$

where $\Delta t$ represents the time step, $\Delta x$ denotes the cell size and $\vec{f}_{i+\frac{1}{2}}$ is the numerical flux in the interface between cells $i$ and $i+1$. The latter represents a numerical approximation of the physical mass and momentum exchange across the interface.

Upwind discretization of the bed slope term $\vec{s}$ (Vázquez-Cendón 1999) introduces a nonconservative contribution in the momentum equation, represented as numerical source term $\vec{s}_{i}$, and a conservative contribution to the mass equation, associated to the interface and grouped in the numerical flux $\vec{f}_{i+\frac{1}{2}}$. The components of these two vectors are denoted as follows:

$$
\vec{f}_{i+\frac{1}{2}}=\left(\begin{array}{c}
f^{h} \\
f^{q}
\end{array}\right)_{i+\frac{1}{2}}, \quad \vec{s}_{i}=\left(\begin{array}{c}
0 \\
s^{q}
\end{array}\right)_{i}
$$

Any conservative numerical method can be formulated following (19), grouping the conservative contribution from the upwind discretization of the bed slope term in the first component of the numerical flux. This becomes important when solving the transport of a solute so that a physical coupling of the two methods is required in those cases.
This procedure is illustrated presenting the contribution to the mass equation from the upwind discretization of the bed slope term in the numerical scheme of Roe (Roe 1986). In the case of supercritical flow this contribution is nil but, when considering subcritical flow, the following can be written:

$$
f_{i+\frac{1}{2}}^{h}={ }^{R o e} f_{i+\frac{1}{2}}^{h}-\frac{1}{2} \sqrt{g \frac{\left(h_{i}+h_{i+1}\right)}{2}}\left(z_{i+1}-z_{i}\right)
$$

The additional resolution of the passive transport of a solute is considered, described by the following conservation law:

$$
\partial_{t} m+\partial_{x}\left(\frac{q m}{h}\right)=0
$$

where $m=m(x, t)$ represents the solute mass and $\phi=m / h$ the associated concentration.

The technique to couple first order methods for the resolution of passive transport and water flow is presented. The proposed method maintains the form of the shallow water scheme (18) extending the vectors of conserved variables $\vec{u}_{i}$, numerical flux $\vec{f}_{i+\frac{1}{2}}$ and source term $\overrightarrow{s_{i}}$ as follows:

$$
\vec{u}_{i}=\left(\begin{array}{c}
h \\
q \\
m
\end{array}\right)_{i}, \vec{f}_{i+\frac{1}{2}}=\left(\begin{array}{c}
f^{h} \\
f^{q} \\
f^{m}
\end{array}\right)_{i+\frac{1}{2}}, \vec{s}_{i}=\left(\begin{array}{c}
0 \\
s^{q} \\
0
\end{array}\right)_{i}
$$

All the discrete approximations in (22) are already defined except the numerical flux of solute mass $f_{i+\frac{1}{2}}^{m}$. This term is estimated using the method proposed by Latorre et al. (Latorre and García-Navarro 2009), based on the physical properties of the mixing of two fluids with different concentrations. The numerical flux $f_{i+\frac{1}{2}}^{h}$ introduces variations of the water mass in the cells $i$ and $i+1$ that are interpreted as physical exchanges of water volumes through the interface. Assuming that the transported water keeps the concentration of the cell of origin, the following expression for the numerical flux of solute mass is obtained:

$$
f_{i+\frac{1}{2}}^{m}=f_{i+\frac{1}{2}}^{h} \phi_{i+\frac{1}{2}}
$$

where the concentration $\phi_{i+\frac{1}{2}}$, dependig on the sign of the water mass numerical flux, is calculated as fol- 
lows:

$$
\phi_{i+\frac{1}{2}}= \begin{cases}\left(\frac{m}{h}\right)_{i} & \text { if } f_{i+\frac{1}{2}}^{h}>0 \\ \left(\frac{m}{h}\right)_{i+1} & \text { if } f_{i+\frac{1}{2}}^{h}<0\end{cases}
$$

Once the numerical solute mass flux is defined, the expression of the solute numerical scheme is:

$$
m_{i}^{t+\Delta t}=m_{i}^{t}-\frac{\Delta t}{\Delta x}\left(f_{i+\frac{1}{2}}^{m}-f_{i-\frac{1}{2}}^{m}\right)
$$

The presented method solves the solute transport in first order of approximation coupled to any numerical scheme for the resolution of the water flow, ensuring a physical behavior (denoted as $P$ property) of the concentration field associated with the solute: preservation of a constant concentration state and monotone behavior. The formulation (18), for the water mass numerical flux, warrants this behavior also in presence of source terms including the effect of the upwind discretization in the passive transport.

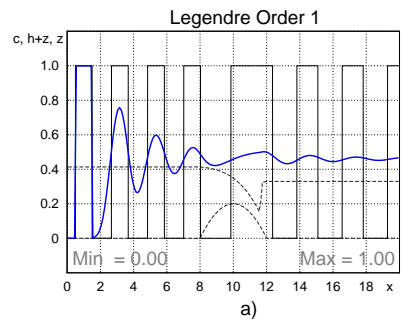

Legendre Order 5

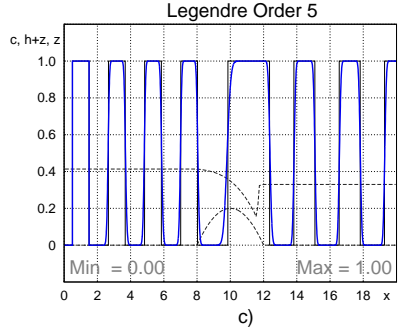

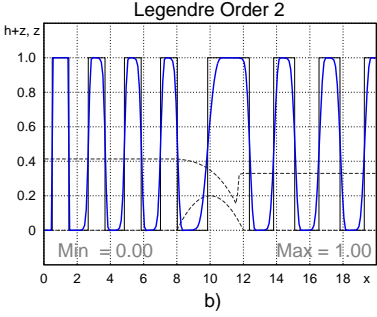

Legendre Order 10

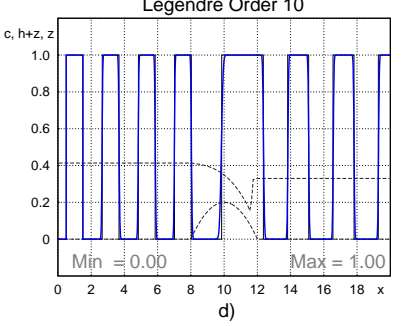

Figure 3: Numerical results and exact solution of solute transport in test case 3 . Water flow is solved using a TVD second order method and solute transport using Legendre schemes of order of approximation 1, 2, 5 and $10 .(\mathrm{t}=40, \mathrm{CFL}=0.9,200$ cells $)$.

The coupling technique is extended to high order Legendre methods computing first, from the water mass numerical flux, $f_{i+\frac{1}{2}}^{h}$, the volume that is transferred through every interface in one time step. This amount is represented as a proportion of the total volume of water contained in each cell at the initial time in the region near the wall. The water volumes are assumed to move through the interface, complying with the net transfer described by the numerical flux.
Two different volumes, corresponding to two depths, are obtained in each cell, whose sum corresponds to the volume calculated by the water flow scheme. The volumes are then assumed to evolve without mixing, adapting their limits and depth to reach the final average state in each cell. This process, whose details can be found in (Latorre 2011), determines the conservative transformation to be applied to the solute mass in the Legendre schemes.

To illustrate the procedure, the formulation considering solute mass transport in a one-dimensional domain and positive water mass numerical fluxes is presented:

$$
\vec{m}_{i}^{t+\Delta t}=\boldsymbol{A} \vec{m}_{i-1}^{t}+\boldsymbol{B} \vec{m}_{i}^{t}
$$

where the matrices $\boldsymbol{A}$ and $\boldsymbol{B}$ depend on the following dimensionless numbers:

$$
c_{1}=\frac{\Delta t f_{i-\frac{1}{2}}^{h}}{\Delta x h_{i-1}^{t}}, c_{2}=\frac{\Delta t f_{i-\frac{1}{2}}^{h}}{\Delta x h_{i}^{t+\Delta t}}, c_{3}=\frac{\Delta t f_{i+\frac{1}{2}}^{h}}{\Delta x h_{i}^{t}}
$$

The proposed scheme (26) solves the passive transport of a solute in the water flow combining the Legendre scheme of arbitrary order of approximation, for the calculation of the transport, and any method for the resolution of the shallow water equations. This coupled method ensures the physical behavior of the concentration field ( $P$ property). This condition also holds in presence of source terms thanks to the formulation (18), that includes the effect of upwind discretization in the transport.

\subsection{Case 3: Transport in a channel}

The transport of a set of solute pulses in a $20 \mathrm{~m}$ long rectangular channel is considered (Fernández-Nieto and Narbona-Reina 2008). The bottom of the channel contains a parabolic profile given by the expression:

$$
z(x)=\max \left(0.2-0.05(x-10)^{2}, 0\right)
$$

The water flow is characterized by a constant discharge, $q=0.18 \mathrm{~m}^{2} / \mathrm{s}$, and a constant water depth at the outlet, $h=0.33 \mathrm{~m}$. Under these boundary conditions, the flow is subcritical at the entrance, is accelerated to change regime in the slope of the obstacle and then becomes subcritical again, showing a hydraulic jump in the transition. The instantaneous injection of solute is performed every five seconds setting the concentration of cells that meet $0.5<x<1.5$ to unit value. 
The steady flow in the channel is computed with a second order TVD numerical scheme (Burguete and García-Navarro 2001) using a regular division of the domain in 200 cells and a time step according to the condition $C F L=0.9$. The transport of the discontinuous profiles of solute mass is coupled by means of (26) using Legendre schemes of order of approximation 1, 2, 5 and 10.

Results for the simulation time $t=40 \mathrm{~s}$ are shown in Figure 3. In each graph the exact solution (continuous black line) and the numerical results (continuous blue line) of the concentration field are represented. The numerical concentration has been obtained as the ratio of the sub-cell solute mass distribution between the constant water depth value supplied by the second order flow scheme. The presented coupled method solves the transport of the solute preserving the $P$ property in presence of bed slope terms and through the hydraulic jump.

\section{CONCLUSIONS}

A method for the simulation of the passive transport of a solute based on Legendre polynomials has been presented. The properties of this technique have been studied and compared with the ADER methodology solving the transport of a Gaussian profile using orders of approximation from 1 to 20. Legendre schemes are more efficient than ADER methods in the resolution of this problem due to the polynomial subcell resolution.

In a second part, a modular technique has been introduced to couple Legendre schemes to any conservative method used for the resolution of the shallow water equations. The coupling technique has been applied to solve the transport of a set of pulses in a steady channel flow including bed level variations, water depth discontinuities and different regimes. Results show the ability of this technique to ensure a physical behavior of the concentration field.

\section{ACKNOWLEDGMENTS}

This research work has been partially funded by the Spanish Ministry of Science and Education under research project CGL2005-07059-C02-02.

\section{References}

Bermudez, A. and M. Vázquez-Cendón (1994). Upwind methods for hyperbolic conservation laws with source terms. Computers and Fluids 23(8), 1049 - 1071.

Burguete, J. and P. García-Navarro (2001). Efficient construction of high-resolution TVD conservative schemes for equations with source terms: application to shallow water flows. International Journal for Numerical Methods in
Fluids 37(2), 209-248.

Burguete, J., J. Murillo, and P. García-Navarro (2008). Preserving bounded and conservative solutions of transport in one-dimensional shallow-water flow with upwind numerical schemes: Application to fertigation and solute transport in rivers. International Journal for Numerical Methods in Fluids 56(9), 17311764.

Dumbser, M. and M. Kaeser (2007). Arbitrary high order non-oscillatory finite volume schemes on unstructured meshes for linear hyperbolic systems. Journal of Computational Physics 221(2).

Fernández-Nieto, E. and G. Narbona-Reina (2008). Extension of WAF Type Methods to Non-Homogeneous ShallowWater Equations with Pollutant. Journal of Scientific Computing 36, 193-217.

Hilbert, D. (1912). Grundzüge einer allgemeinen theorie der linearen integralgleichungen. $B . G$. Teubner, Leipzig.

Latorre, B. (2011). Simulación eficiente del transporte pasivo en flujos de superficie libre. $\mathrm{Ph} . \mathrm{D}$. thesis, University of Zaragoza, Spain.

Latorre, B. and García-Navarro (2009). An efficient and conservative model for solute transport in unsteady shallow water flow. In P. López-Jiménez (Ed.), Proceedings of the Int. Workshop on Environmental Hydraulics, pp. 114-119.

Latorre, B. and P. García-Navarro (2011). Accurate and efficient simulation of transport in multidimensional flow. International Journal for $\mathrm{Nu}$ merical Methods in Fluids 65(4), 405-431.

Murillo, J., P. García-Navarro, and J. Burguete (2009). Conservative numerical simulation of multi-component transport in two-dimensional unsteady shallow water flow. Journal of Computational Physics 228(15), 5539-5573.

Roe, P. L. (1986). Upwind differencing schemes for hyperbolic conservation laws with source terms. Proceedings of the 1st international Congress on Hyperbolic Problems, 41-51.

Rusanov, V. (1963). Characteristics of the general equations of gas dynamics. Zhurnal Vychislitelnoi Mathematiki Mathematicheskoi Fiziki 3, 508-527.

Smolarkiewicz, P. (1982). The multi-dimensional Crowley advection scheme. Mon. Wea. Rev. 113, 1050-1065. 
Toro, E., R. Millington, and L. Nejad (2001). Towards very high order Godunov schemes. Kluwer/Plenum Academic Publishers.

Vázquez-Cendón, M. (1999). Improved treatment of source terms in upwind schemes for the shallow water equations in channels with irregular geometry. Journal of Computational Physics 148(2), 497 - 526. 